\title{
REVIEW
}

\section{Investigation of measured and predicted resting energy needs in adults after spinal cord injury: a systematic review}

\author{
AN Nevin ${ }^{1,3}$, J Steenson ${ }^{1,3}$, A Vivanti ${ }^{1}$ and IJ Hickman ${ }^{1,2}$
}

Background: Accurate estimation of energy needs is vital for effective nutritional management of individuals with spinal cord injury (SCI). Inappropriate energy prescription after SCI can compound the rates of malnutrition or obesity, increase the risk of complications and negatively influence outcomes. Energy requirements following $\mathrm{SCl}$ are not well understood, and there is currently no universally accepted method of estimating energy needs in clinical practice.

Study design: This is a systematic literature review.

Objectives: The objectives of this study were to investigate and compare the measured resting energy needs of adults with SCl across different phases of rehabilitation, and to identify appropriate energy prediction equations for use in $\mathrm{SCl}$.

Setting: This study was conducted in Australia.

Methods: MEDLINE, EMBASE and CENTRAL databases were searched for studies published between 1975 and April 2015 , identifying 298 articles. Full articles in English language of adults with SCl who were fasted for a minimum of 8 hours before undergoing indirect calorimetry to measure resting energy expenditure (REE) for at least 20 min were selected. On the basis of the inclusion criteria, 18 articles remained for data extraction. One author extracted information from all articles, and inter-rater reliability was tested in five articles.

Results: REE across three phases of injury was assessed: acute, sub-acute and chronic. Few studies $(n=2)$ have investigated REE in the acute and sub-acute injury stages of SCl recovery. The factors influencing chronic energy needs in $\mathrm{SCl}$ patient populations are many and varied, and a valid predictive equation for use in $\mathrm{SCl}$ remains elusive.

Conclusion: Indirect calorimetry remains the only accurate assessment of REE for health practitioners working with patients after SCI. Spinal Cord (2016) 54, 248-253; doi:10.1038/sc.2015.193; published online 22 December 2015

\section{INTRODUCTION}

Physiological factors create challenges when estimating the energy needs of people with a spinal cord injury (SCI) in clinical practice. SCI is initially associated with catabolic conditions of trauma such as spinal shock and patients may be at risk of weight loss, malnutrition and pressure injuries. ${ }^{1}$ In the long term, SCI results in marked body composition changes, specifically muscle atrophy below the level of injury and higher relative fat mass compared with people without $\mathrm{SCI},{ }^{1-3}$ which may contribute to decreases in resting energy expenditure (REE). In an environment of reduced mobility, energy needs are further reduced, and there is an increased risk of weight gain and obesity-related chronic diseases. ${ }^{4,5}$

To prescribe an appropriate daily energy intake, an individual's total daily energy expenditure (TDEE) must be determined. An individual's TDEE comprises three components, two of which may be considerably affected by SCI. Basal metabolic rate (BMR) is the minimum energy required for the basal processes of life, and it contributes the majority $(\sim 70 \%)$ of TDEE in a healthy person. ${ }^{6}$ In the clinical literature, the term BMR is often used interchangeably with REE, and for the purpose of this review REE is defined as the energy expended in an awake, alert individual in a rested position following an overnight fast (post-absorptive state), in a thermoneutral ambient environment and before any physical activity. As REE is predominantly related to body size and composition, it is likely to vary widely across the lifetime following SCI. ${ }^{4,7}$ The second component is activity energy expenditure (AEE), which is the energy used above resting needs for any incidental or planned physical movement, and mental and emotional stimulation, and it can account for $~ 20 \%$ of TDEE in active individuals. ${ }^{6}$ The impact of SCI on AEE will vary considerably on the basis of the injury type, with complete or partial loss of motor function greatly reducing the amount of energy that can be expended through any physical movement. The third component is the thermic effect of food (TEF), which is the energy expended because of digestion of food, and it generally accounts for $\sim 10 \%$ of TDEE. ${ }^{6}$ There is no evidence to suggest that this is influenced by SCI. ${ }^{7}$

As REE comprises the greatest proportion of TDEE, much attention is placed on its accurate measurement. Indirect calorimetry is considered the gold standard for assessing REE, but it is typically confined to the research setting. In clinical practice where accurate assessment methodologies may not be readily available, predictive equations are often used as a surrogate. The most commonly used predictive energy equations in general clinical practice are not reliable

${ }^{1}$ Department of Nutrition and Dietetics, Princess Alexandra Hospital, Woolloongabba, Queensland, Australia and ${ }^{2}$ Mater Research Institute University of Queensland, Translational Research Institute, Brisbane, Queensland, Australia

${ }^{3}$ These authors contributed equally to this work.

Correspondence: AN Nevin, Department of Nutrition and Dietetics, Princess Alexandra Hospital, Ipswich Rd, Woolloongabba, 4102 Queensland, Australia.

E-mail: Amy.Nevin@health.qld.gov.au

Received 20 November 2014; revised 24 August 2015; accepted 21 September 2015; published online 22 December 2015 
in SCI given that they are derived from uninjured populations. ${ }^{8,9}$ Current recommendations for predicting REE in SCI rehabilitation involve the use of a ratio equation, ${ }^{10}$ which is based on a single study that was undertaken more than 20 years ago in hospitalised rehabilitating patients. ${ }^{8}$ It is unclear whether the development of more widely validated equations has progressed since this time.

The objectives of this review were to investigate and compare the objectively measured REE of patients with SCI across different phases of injury, and to identify and appraise energy prediction equations that are appropriate for use in patients with SCI.

\section{MATERIALS AND METHODS}

\section{Database search}

All studies were retrieved through a comprehensive literature search. The search was conducted through Ovid via MEDLINE, EMBASE and CENTRAL using the following limits: text word search, English language only, humans, adult participants only (19 years or more). Search terms were selected from known articles relevant to the subject heading. Search terms included the following: SCI; spinal cord injury; spinal cord injuries; spinal $\operatorname{cord}^{*}$; spinal injur ${ }^{*}$; paraplegia; tetraplegia; quadriplegia; REE; resting energy expenditure; BMR; basal metabolic rate; BEE; basal energy expenditure; RMR; resting metabolic rate; ${ }^{\star}$ energy expenditure; energy expend ${ }^{*}$; energy expenditure*; estimated energy ${ }^{*}$; energy estimation; energy require*; energy requirement; energy need ${ }^{*}$; calori $^{*}$; calorimetry. Databases were searched for literature published between 1975 and April 2015.

\section{Exclusion criteria}

Primary article exclusion criteria included the following: non-English language, those without abstracts, full articles unable to be retrieved through extensive database searches and library services $(n=4)$, review articles and studies in which REE and/or study population characteristics were not adequately specified and studies including participants without SCI. If studies by the same authors were identified and found to include similar participants and mean REE values $(n=4),{ }^{11-14}$ the study with the largest sample size was included. Case studies were excluded because of the low level IV evidence, ${ }^{15}$ and studies with adolescent data were excluded as the review was confined to the adult

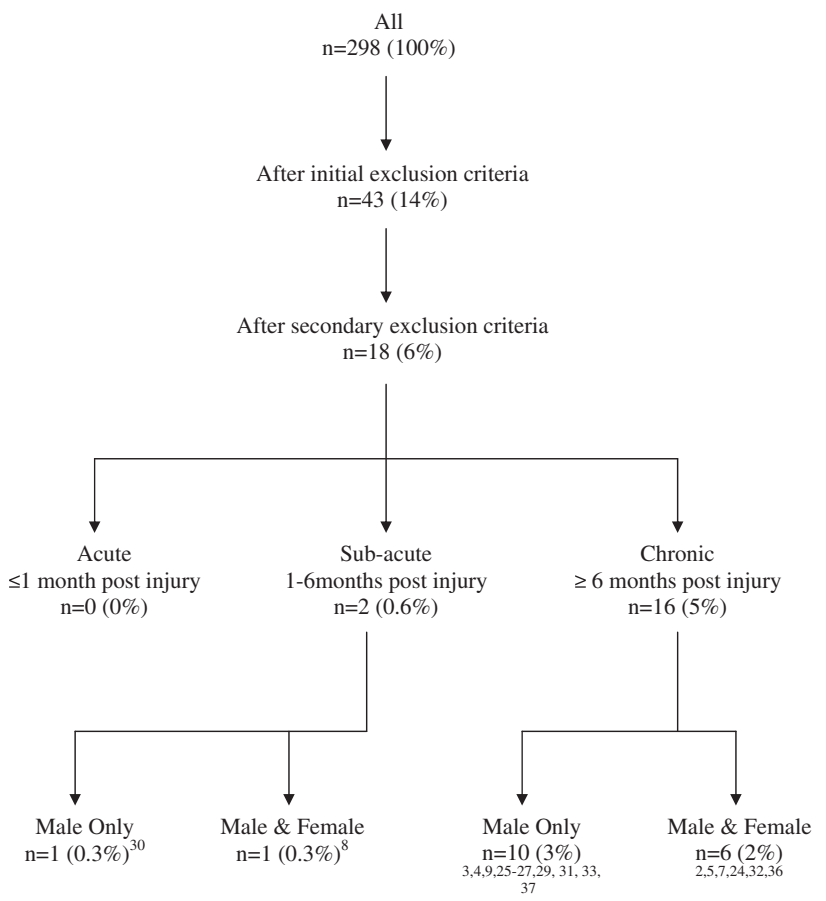

Figure 1 Article selection for measured and predicted energy needs after spinal cord injury. population only. A secondary exclusion criteria was applied when methodology may have compromised data quality. The REE protocol must have been clearly defined, participants needed to be fasted for at least 8 hours ${ }^{16,17}$ to remove effects of diet-induced thermogenesis and REE must be measured for at least $20 \mathrm{~min}$ to ensure attainment of steady-state gas exchange. ${ }^{16,17}$ Studies were included if they met the testing conditions, whether or not the measurement was taken immediately upon waking or after waking and travelling from home to the site of testing with a resting period before the commencement of testing. Studies were also included irrespective of which terminology was used to define test output: for example, REE or BMR.

\section{Charting data according to key concepts}

Articles were grouped according to time post injury. ${ }^{18}$ Participants less than 1 month following injury were grouped as 'acute' because this typically represents a period of catabolic stress brought about by surgical intervention, ${ }^{19}$ increased prevalence of complications ${ }^{20}$ and negative nitrogen balance coinciding with weight loss. ${ }^{21}$ Patients between one and six months after injury were grouped as 'sub-acute.' For many, this is a time of intensive rehabilitation aimed at optimising quality of life and independence, ${ }^{22}$ and it may represent a period of ongoing negative nitrogen balance and weight instability. ${ }^{21}$ Those greater than 6 months after injury were grouped in the 'chronic' phase, as they are more likely to be medically stable, undergoing final stages of rehabilitation or have returned home. ${ }^{22}$

Not all studies reported SCI using the American Spinal Injury Association (ASIA) impairment scale (AIS). ${ }^{23}$ Injuries referred to as 'complete' were interpreted as motor complete, corresponding with AIS A or B injuries. 'Incomplete' injuries were interpreted as motor incomplete, corresponding with AIS C or D injuries.

\section{Inter-rater reliability testing}

Inter-rater reliability was assessed in a randomly selected sample $(12 \%, 5 / 43)$ of articles remaining after the first exclusion criteria were applied. Data were extracted and performed in a blinded manner by a second researcher. Cohen's kappa coefficient was used to check agreement with the original researcher $(\kappa=1.0(100 \%))$, indicating perfect agreement.

\section{Statistical analysis}

To remain consistent with published literature, REE is reported as mean \pm s.d. For studies that present findings as mean \pm s.e.m., the s.e.m. was converted to a s.d. using the following equation:

$$
\text { s.d. }=\text { s.e. } \times \sqrt{\mathrm{n}}
$$

Where s.d. is the standard deviation, s.e.m. is the standard error and $n$ is the sample size. Several studies reported findings by subgroup only, such as male and female or paraplegic and tetraplegic, ${ }^{3,24-27}$ and it failed to report a mean for the cohort. To enable ease of comparison between studies, subgroup means were combined and a weighted group mean and standard deviation was calculated for each study using the following equation:

$$
\bar{\chi}_{w}=\sum_{i=1}^{s}\left(\frac{n_{i}}{N}\right) \times \bar{\chi}_{i}
$$

where $w$ is the weighted mean, $s$ is the number of subgroups, $n$ is the subgroup size and $N$ is the cohort size. For consistency, mean REE for all studies is expressed as kcal per day. A conversion factor of 0.239 was used to convert $\mathrm{kJ}$ to $\mathrm{kcal}^{28}$ whereas REE per min was converted to REE per day using a conversion factor of 1440 .

\section{RESULTS}

The literature search returned 298 articles. After applying initial exclusion criteria, 43 articles $(14 \%, 43 / 298)$ remained. Secondary exclusion criteria for scientific quality removed 12 studies in which patients were fasted for less than 8 hours; eight studies that measured REE for less than $20 \mathrm{~min}$; and five studies with insufficient information regarding the REE testing protocol used (Figure 1).

The remaining 18 articles $(6 \%, 18 / 298)$ were then analysed (Supplementary Information-Table 1). Three of the 18 studies did 
Table 1 Reported resting energy expenditure

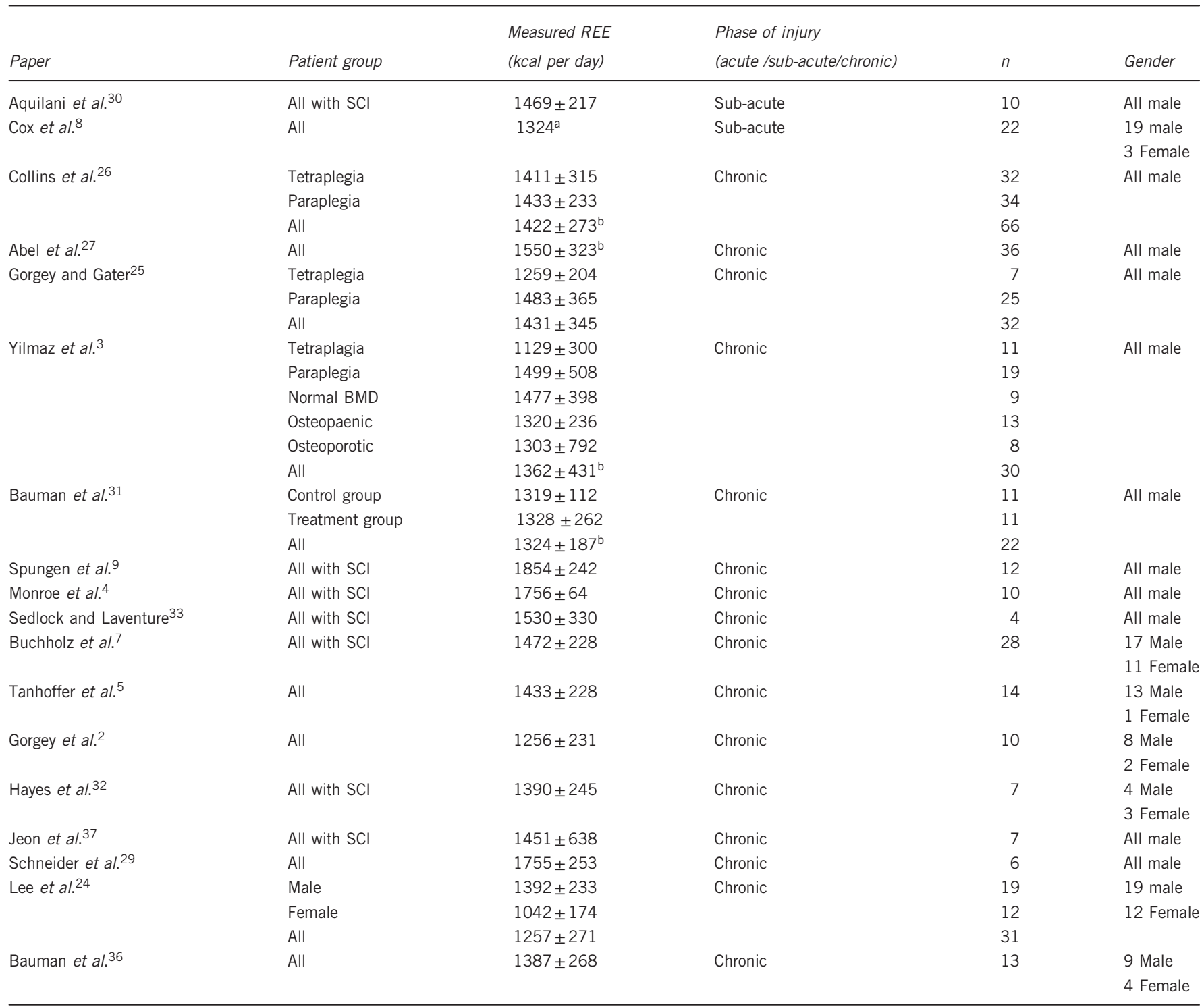

Abbreviations: BMD, bone mineral density; SCI, spinal cord injury.

as.d. not reported.

bWeighted mean and s.d.

not report the time since injury but were grouped in the chronic phase. This was because of statements such as 'subjects were physically active', 29 'five of the paraplegic subjects participated in recreational basketball $^{29}$ or study methodology that required participants to complete domestic activities in their home, ${ }^{24}$ suggesting that participants were independent, not undergoing inpatient rehabilitation and likely greater than six months post injury.

\section{Measured REE}

No identified studies assessed REE in the acute phase post injury. Two studies assessed REE in the sub-acute phase (1-6 months post injury), where mean REE ranged from 1324 to $1469 \mathrm{kcal}$ per day. The remaining 16 studies measured REE at least 6 months post injury, the chronic phase of SCI rehabilitation (Figure 1) where mean REE ranged from 1256 to $1855 \mathrm{kcal}$ per day (Table 1). These findings prevent valid comparisons of REE across different phases of injury.
Furthermore, all studies reported cross-sectional data with no observational studies examining longitudinal assessment of REE after SCI.

Study participants were predominantly male, with $61 \%(11 / 18)$ of studies conducted in male-only populations and the remaining $39 \%$ (7/18) performed in mixed-gender populations. Complete injuries (AIS A or B) were the most common SCIs reported in participants (59\%, 200/337) (Supplementary information-Table 1). Five (28\%, $5 / 18$ ) studies did not report injury completeness or AIS classification.

Some authors compared REE between SCI subgroups such as paraplegic and tetraplegic, male and female (Table 1). Both Yilmaz et al. and Gorgey et al. report a difference in REE between tetraplegic and paraplegic participants of 370 and $224 \mathrm{kcal}$ per day, respectively, 3,25 whereas Collins et al. found there to be no difference. ${ }^{26}$ Any comparisons between men and women showed differences between genders similar to that expected in the general population. Differences in measured REE were also observed between other SCI subgroups in the study by Yilmaz ${ }^{3}$ where REE was found to correlate with lower 
Table 2 Predicted versus measured resting energy expenditure

\begin{tabular}{|c|c|c|c|c|}
\hline Paper & Equation used ${ }^{a}$ & $\begin{array}{c}\text { Predicted REE } \\
\text { (kcal per day) }\end{array}$ & $\begin{array}{c}\text { Measured REE } \\
\text { (kcal per day) }\end{array}$ & $\begin{array}{c}\% \text { overestimate }(+) \text { or } \\
\text { underestimate }(-)\end{array}$ \\
\hline Aquilani et al. ${ }^{30}$ & Harris Benedict & $1530 \pm 210$ & $1469 \pm 217$ & +4 \\
\hline \multirow[t]{4}{*}{ Cox et al. ${ }^{8}$} & Harris Benedict $\times 1.2$ & $2030 \pm 192$ & $1589 \pm 319^{b}$ & +28 \\
\hline & Harris Benedict $\times 1.75$ & $2960 \pm 286$ & & +86 \\
\hline & Quebbeman & $1589 \pm 159$ & & 0 \\
\hline & Spanier and Shizgal & $3056 \pm 0$ & & +92 \\
\hline Bauman et al. ${ }^{31}$ & Harris Benedict & $1747 \pm 143$ & $1324 \pm 187^{c}$ & +32 \\
\hline Sedlock and Laventure 33 & Cunningham & $1718 \pm 150$ & $1530 \pm 330$ & +12 \\
\hline Buchholz et al. ${ }^{7}$ & Schofield & $1553 \pm 261$ & $1472 \pm 228$ & +6 \\
\hline \multirow[t]{2}{*}{ Hayes et al. ${ }^{32}$} & Harris Benedict & $1601 \pm 360$ & $1390 \pm 245$ & +15 \\
\hline & Authors own & $1365 \pm 277$ & & -2 \\
\hline
\end{tabular}

Abbreviation: REE, resting energy expenditure.

asee Table 3 for prediction equations.

biffers from measured REE displayed in Table 2; the authors applied a $20 \%$ activity factor to enable comparison against listed energy prediction equations.

'Weighted mean and s.d.

limb bone mineral density (Table 1). The s.d. between all studies varied greatly because of small study numbers and/or heterogeneity in patient groups such as combined genders and injury levels.

\section{Predictive equations}

Measured REE was compared with a predictive equation in six $(33 \%$, 6/18) articles (Table 2). Predictive equations tested in these validation studies included the following: Harris Benedict, ${ }^{8,30-32}$ Schofield, ${ }^{12}$ Quebbeman, ${ }^{8}$ Spanier and Schizgal ${ }^{8}$ and Cunningham, ${ }^{33}$ which are all derived from populations without SCI. One study compared REE with an equation developed by the authors ${ }^{32}$ (Table 3 ).

Three of the four studies that compared the Harris Benedict equation with measured $\mathrm{REE}^{8,31,32}$ found the equation to overestimate REE by up to $30 \%$ compared with indirect calorimetry measurements. When Cox applied an injury factor of 1.75 (as recommended in previously published work for patients in states of mild-moderate catabolism), ${ }^{34}$ the overestimation of REE increased to $90 \%$. Although the study by Aquilani was the only one to find measured REE similar to predicted REE using the Harris Benedict equation, the authors highlighted that the study populations were likely to be hypermetabolic because of the presence of pressure injuries and urinary tract infections. ${ }^{30}$

Cox found no difference when comparing measured REE with predicted REE using the Quebbeman equation at the group level; however, the correlation between measured and predicted REE was low (Pearson's correlation coefficient $=0.36){ }^{8}$ Hayes was the only other study to find measured REE similar to predicted REE, using an equation that relied on measurements from dual-energy X-ray absorptiometry (DXA). ${ }^{32}$

\section{DISCUSSION}

Accurate assessment of energy needs is paramount to prescribing appropriate nutritional support following SCI. Achieving and maintaining a healthy weight for individuals with SCI is vital to reduce the risk of developing cardiovascular disease, type 2 diabetes and other weight-related chronic conditions. Findings from this systematic review emphasise the absence of data surrounding energy requirements in the first 6 months following injury, while highlighting the diversity of factors that impact energy needs across the SCI rehabilitation journey. The predictive energy equations used in studies included in this review were largely derived from uninjured populations, failed to take into account these influencing factors and therefore overestimated REE in SCI patients.

It has been proposed that the energy needs of people with SCI change over time, ${ }^{2}$ which is similar to that shown in traumatic brain injury. ${ }^{35}$ The REE of an individual with SCI is thought to be raised in the acute phase of injury as the body goes through spinal shock ${ }^{1}$ and then decrease to below energy needs before injury (as measured by matched controls). ${ }^{12,26,36,37}$ This review found only two studies that measured REE within the first 6 months after SCI (mean $\mathrm{REE}=1324-1469 \mathrm{kcal}$ per day), ${ }^{8,30}$ and it was unable to capture the hypothesised increased energy needs in the 'acute phase,' compared with the decreased energy needs in the 'chronic phase' of SCI. The lack of data may be a result of contraindications to performing indirect calorimetry in acute care settings such as renal replacement therapy, supplemental oxygen, tracheostomies and chest tubes/drains. ${ }^{6}$ Further, ventilated patients require specific models of indirect calorimeters that may be unavailable in some facilities. Regardless, it remains a crucial aspect of SCI rehabilitation that remains unclear and deserves greater focus in future research efforts.

The variation in measured REE across chronic injury studies was significant (1256-1854 kcal per day), and it demonstrates the importance of identifying the role of confounding factors when assessing energy needs in this often heterogeneous patient population. The well-established effect of gender on energy requirements is maintained after SCI, with REE over $300 \mathrm{kcal}$ per day higher in men. The lowest REE values found by Gorgey et al. ${ }^{2}$ and Lee et al. ${ }^{24}$ ( $1256 \mathrm{kcal}$ per day) may be explained by the combined gender data and most study participants having motor complete injuries. People with motor complete injuries are thought to have lower energy needs compared with those with motor incomplete injuries because of greater reductions in lean body mass. ${ }^{25,26}$

The loss of lean body mass following SCI is obligatory, ${ }^{21}$ and it is also influenced by the neurological level of SCI. ${ }^{38}$ Patients with tetraplegia often experience a greater loss of muscle mass compared with those with paraplegia, ${ }^{38}$ which appears to reduce energy expenditure further. This was demonstrated in the studies by Yilmaz and Gorgey, in which REE was up to $370 \mathrm{kcal}$ per day lower in tetraplegic compared with paraplegic patients. With previous work suggesting that REE does not differ between paraplegic and ablebodied subjects after adjustment for fat-free mass and body cell mass, ${ }^{7}$ further research on the role of body composition as a predictor of energy needs may be worthwhile. 
Table 3 Energy prediction equations

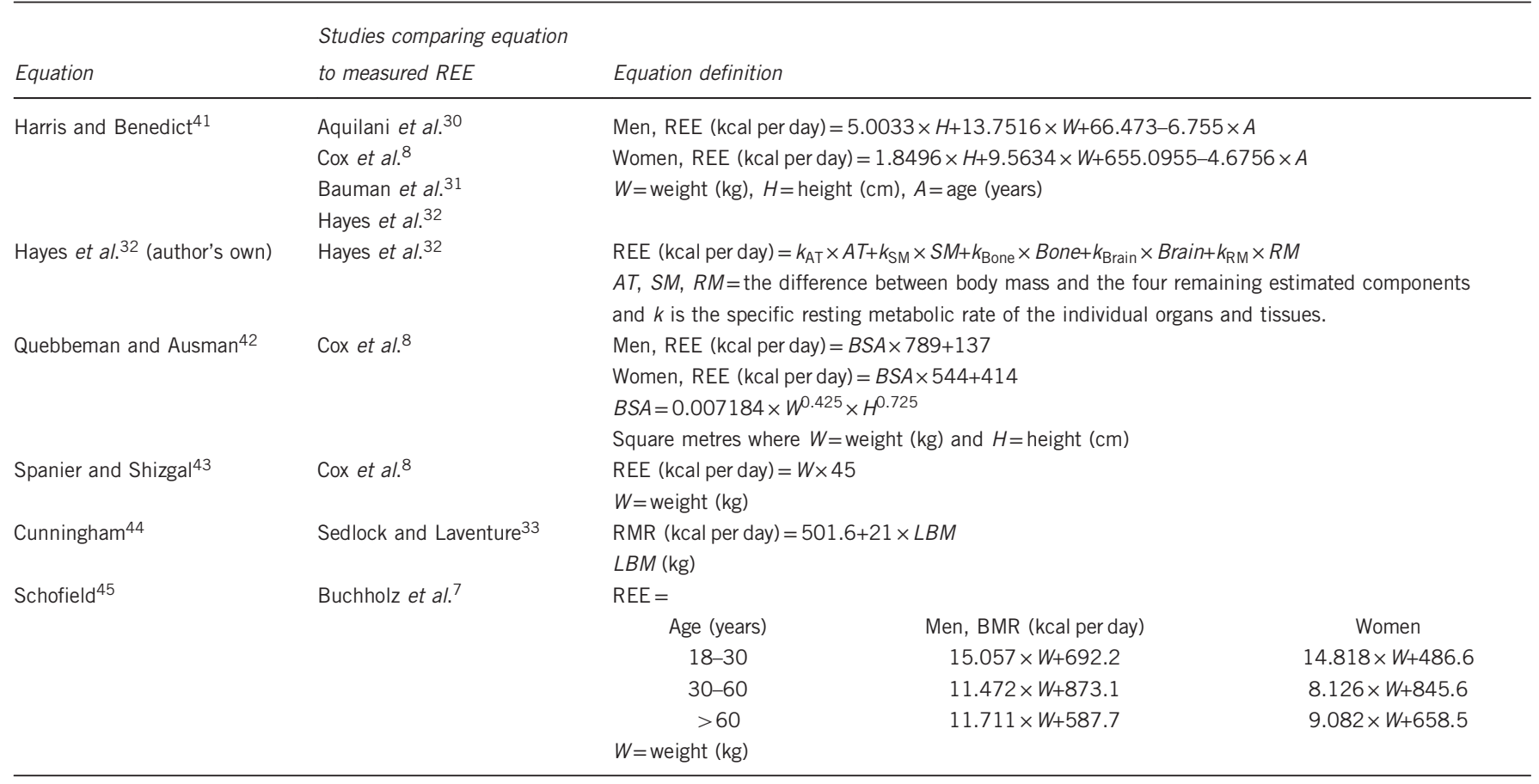

Abbreviations: AEE, activity energy expenditure; AT, adipose tissue; BMR, basal metabolic rate; BSA, body surface area; LBN, lean body mass; RM, residual mass; SM, skeletal muscle mass.

In addition to individual variation associated with gender, injury level, injury completeness and lean body mass, there may be other factors influencing REE in SCI, which evolve over the course of rehabilitation. Bone mineral density was found to correlate with REE in the study by Yilmaz et al., ${ }^{3}$ whereas Bauman found that REE and lean tissue mass increased significantly in hypogonadal men with SCI receiving testosterone replacement therapy. ${ }^{31}$ Anti-spasticity medications commonly prescribed in SCI have been shown to suppress REE in non-injured populations, ${ }^{39}$ and common secondary complications such as pressure injuries and urinary tract infections may increase energy needs. ${ }^{30}$ The complexity of influencing factors, which may be unique to each individual, increases the difficulty of developing a valid predictive equation for broad clinical use in those with SCI.

Attempts to validate predictive equations derived from uninjured populations were unsuccessful in the studies of SCI patients included in this review, with some equations overestimating REE by up to $90 \%$. The equation developed by Hayes was the only study to find predicted REE similar to measured REE, using DXA measurements and equations derived from these. Although the study was small and the equation is yet to be validated in a larger population, findings lend further merit to the role of body composition as a key predictor of REE after SCI.

\section{Study strengths and limitations}

The main limitation of this review is that most identified studies included small sample sizes with often incomplete specification of study populations regarding injury level, completeness, time since injury and body weight. The search strategy to limit articles from English language studies only may have led to search bias, and the review was restricted to adult participants only. Many authors investigate the effects different activities have on energy expenditure in the SCI population; however, many fail to report a baseline REE and could not be included in the review. Further, data reported as kcal per day (not kcal per kg per day) somewhat limits comparisons between investigated populations
For translation of REE data into clinical energy prescription, daily activity data are required, and this was not included here. Although there is a multitude of literature examining energy expenditure during physical activity after SCI, it is unclear whether these data can yet translate to activity factors capable of complimenting REE measurements in clinical practice.

Study strengths include the comprehensive nature of the systematic literature review and the strict inclusion criteria. The secondary exclusion criteria enhanced scientific quality. We confirmed consistency of data extraction through a blinded sub-study of five randomly selected published articles of those retrieved.

\section{Areas for future research}

Longitudinal studies tracking energy needs from acute injury through to chronic SCI in larger cohorts are needed to accurately demonstrate how energy needs change over time after SCI. Monitoring energy needs longitudinally during the development and healing of pressure ulcers and in those undergoing surgical intervention or with active infections is also paramount to better inform the application of injury factors across the course of SCI rehabilitation. Future efforts to develop a predictive energy equation for SCI should closely consider the role of body composition as a key predictor of REE, and should this progress establishment of bedside methods of body composition assessment validated for the SCI population is likely to be useful. Investigating the feasibility of introducing indirect calorimetry into standard care of SCI and pursuing lower cost alternatives to current equipment needs is warranted. In addition, there is also a need to accurately and objectively measure physical activity across different SCI levels and AIS grades to inform AEE values. This would aid the development of SCI-specific activity factors, which is a vital component of an energy prediction equation. Such information may also shed light on the possible variations in the contribution of each component of energy expenditure (REE and AEE) on TDEE, which is yet to be clearly established in SCI. 


\section{CONCLUSIONS}

There are limited studies of REE in the acute and sub-acute injury stages of SCI recovery where energy needs may be greater. In the two studies conducted in sub-acute SCI, mean REE ranged from 1324 to $1469 \mathrm{kcal}$ per day. Because of the heterogeneous study cohorts, there was a high degree of variation in REE reported in the chronic phase of SCI, where mean REE ranged from 1256 to 1854 kcal per day. As a result, alterations in short- and long-term energy needs of patients after SCI remain unclear. To date, the progress towards developing a validated predictive energy equation targeted for SCI has been slow and unsuccessful, and indirect calorimetry remains the only accurate assessment of REE for health practitioners working with individuals after SCI.

* The search criteria for this systematic review included articles published until April 2015. A study published beyond this date, which otherwise met remaining search criteria, reported a mean REE of $1467 \pm 229 \mathrm{kcal}$ in men with chronic complete SCI (C5-T10). ${ }^{40}$ This finding remains consistent with the range of values reported in this review for chronic SCI and does not alter the study results or conclusion.

\section{CONFLICT OF INTEREST}

The authors declare no conflict of interest.

1 Dionyssiotis Y. Malnutrition in spinal cord injury: more than nutritional deficiency. J Clin Med Res 2012; 4: 227-236.

2 Gorgey AS, Chiodo AE, Zemper ED, Hornyak JE, Rodriguez GM, Gater DR. Relationship of spasticity to soft tissue body composition and the metabolic profile in persons with chronic motor complete spinal cord injury. J Spinal Cord Med 2010; 33: 6-15.

3 Yilmaz B, Yasar E, Goktepe AS, Onder ME, Alaca R, Yazicioglu K et al. The relationship between basal metabolic rate and femur bone mineral density in men with traumatic spinal cord injury. Arch Phys Med Rehab 2007; 88: 758-761.

4 Monroe MB, Tataranni PA, Pratley R, Manore MM, Skinner JS, Ravussin E. Lower daily energy expenditure as measured by a respiratory chamber in subjects with spinal cord injury compared with control subjects. Am J Clin Nutr 1998; 68: 1223.

5 Tanhoffer RA, Tanhoffer AIP, Raymond J, Hills AP, Davis GM. Comparison of methods to assess energy expenditure and physical activity in people with spinal cord injury. J Spinal Cord Med 2012; 35: 35-45.

6 Garrow JS, James WPT. Human Nutrition and Dietetics. 9th edn Churchill Livingstone Medical Division, New York, NY, USA, 1993.

7 Buchholz AC, McGillivray CF, Pencharz PB. Differences in resting metabolic rate between paraplegic and able-bodied subjects are explained by differences in body composition. Am J Clin Nutr 2003; 77: 371.

8 Cox SA, Weiss SM, Posuniak EA, Worthington P, Prioleau M, Heffley G. Energy expenditure after spinal cord injury: an evaluation of stable rehabilitating patients. J Traum 1985; 25: 419-423.

9 Spungen AM, Bauman WA, Wang J, Pierson JRN. The relationship between total body potassium and resting energy expenditure in individuals with paraplegia. Arch Phys Med Rehab 1993; 74: 965.

10 American Dietetic Association. Spinal Cord Injury (SCI) Evidenced-Based Nutrition Practice Guideline. American Dietetic Association: Chicago (IL), 2009.

11 Yilmaz B, Yasar E, Goktepe S, Alaca R, Yazicioglu K, Dal U et al. Basal metabolic rate and autonomic nervous system dysfunction in men with spinal cord injury. Obesity 2007; 15: 2683-2687.

12 Buchholz AC, McGillivray CF, Pencharz PB. Physical activity levels are low in free-living adults with chronic paraplegia. Obes Res 2003; 11: 563-570.

13 Lee M, Zhu W, Hedrick B, Fernhall B. Estimating MET values using the ratio of HR for persons with paraplegia. Med Sci Sports Exerc 2010; 42: 985-990.

14 Bauman WA, La Fountaine MF, Cirnigliaro CM, Kirshblum SC, Spungen AM. Lean tissue mass and energy expenditure are retained in hypogonadal men with spinal cord injury after discontinuation of testosterone replacement therapy. J Spinal Cord Med 2015; 38: 38-47.

15 National H, Medical Research C. How to Use the Evidence: Assessment and Application of Scientific Evidence. NHMRC: Canberra, 2000.

16 Weir JB. New methods for calculating metabolic rate with special reference to protein metabolism. J Physiol 1949; 109: 1-9.
17 Roffey DM, Byrne NM, Hills AP. Day-to-day variance in measurement of resting metabolic rate using ventilated-hood and mouthpiece \& nose-clip indirect calorimetry systems. JPEN J Parenter Enteral Nutr 2006; 30: 426-432.

18 Academy of Nutrition and Dietetics Evidence Analysis Library. Spinal Cord Injury Toolkit. Academy of Nutrition and Dietetics: Chicago (IL), 2014.

19 Fehlings MG, Rabin D, Sears W, Cadotte DW, Aarabi B. Current practice in the timing of surgical intervention in spinal cord injury. Spine 2010; 35: S166-S173.

20 Grossman RG, Frankowski RF, Burau KD, Toups EG, Crommett JW, Johnson MM et al. Incidence and severity of acute complications after spinal cord injury. J Neurosurg-Spine 2012; 17: 119-128.

21 Rodriguez DJ, Clevenger FW, Osler TM, Demarest GB, Fry DE. Obligatory negative nitrogen balance following spinal cord injury. JPEN J Parenter Enteral Nutr 1991; 15: 319-322.

22 Wolfe DL, Hsieh JTC, Mehta S. Rehabilitaion practices and associated outcomes following spinal cord injury. In: Eng JJ, Teasell RW, Miller WC, Wolfe DL, Townson AF, Hseih JTC et al. (eds). Spinal Cord Injury Rehabiliation Evidence, Version 4.0, 2012. http://www.scireproject.com/rehabilitation-evidence/rehabilitation-practices.

23 Kirshblum SC, Burns SP, Biering-Sorensen F, Donovan W, Graves DE, Jha A et al. International standards for neurological classification of spinal cord injury (Revised 2011). J Spinal Cord Med 2011; 34: 535-546.

24 Lee M, Zhu W, Hedrick B, Fernhall B. Determining metabolic equivalent values of physical activities for persons with paraplegia. Disabil Rehabil 2010; 32: 336-343.

25 Gorgey AS, Gater DR. Regional and relative adiposity patterns in relation to carbohydrate and lipid metabolism in men with spinal cord injury. Appl Physiol Nutr Me 2011; 36: 107-114.

26 Collins EG, Gater D, Kiratli J, Butler J, Hanson K, Langbein WE. Energy cost of physical activities in persons with spinal cord injury. Med Sci Sport Exer 2010; 42: 691-700.

27 Abel T, Platen P, Rojas Vega S, Schneider S, Strüder HK. Energy expenditure in ball games for wheelchair users. Spinal Cord 2008; 46: 785-790.

28 Food and Agriculture Organization (FAO). Food Energy - Methods of Analysis and Conversion Factors FAO Food and Nutrition Paper 77. Report of a Technical Workshop Rome, 3-6 December 2002: Rome, 2003.

29 Schneider DA, Sedlock DA, Gass E, Gass G. VO2peak and the gas-exchange anaerobic threshold during incremental arm cranking in able-bodied and paraplegic men. Eur $J$ Appl Physiol Occup Physiol 1999; 80: 292-297.

30 Aquilani R, Boschi F, Contardi A, Pistarini C, Achilli MP, Fizzotti G et al. Energy expenditure and nutritional adequacy of rehabilitation paraplegics with asymptomatic bacteriuria and pressure sores. Spinal Cord 2001; 39: 437-441.

31 Bauman WA, Cirnigliaro CM, La Fountaine MF, Jensen AM, Wecht JM, Kirshblum SC et al. A small-scale clinical trial to determine the safety and efficacy of testosterone replacement therapy in hypogonadal men with spinal cord injury. Horm Metab Res 2011; 43: 574-579.

32 Hayes M, Chustek M, Wang Z, Gallagher D, Heshka S, Spungen A et al. DXA: potential for creating a metabolic map of organ-tissue resting energy expenditure components. Obes Res 2002; 10: 969-977.

33 Sedlock DA, Laventure SJ. Body composition and resting energy expenditure in long term spinal cord injury. Paraplegia 1990; 28: 448.

34 Rutten P, Blackburn GL, Flatt JP, Hallowell E, Cochran D. Determination of optimal hyperalimentation infusion rate. J Surg Res 1975; 18: 477-483.

35 McEvoy CT, Cran GW, Cooke SR, Young IS. Resting energy expenditure in nonventilated, non-sedated patients recovering from serious traumatic brain injury: comparison of prediction equations with indirect calorimetry values. Clin Nutr 2009; 28: 526-532.

36 Bauman WA, Spungen AM, Wang J, Pierson JRN. The relationship between energy expenditure and lean tissue in monozygotic twins discordant for spinal cord injury. J Rehabil Res Dev 2004; 41: 1.

37 Jeon JY, Steadward RD, Wheeler GD, Bell G, McCargar L, Harber V. Intact sympathetic nervous system is required for leptin effects on resting metabolic rate in people with spinal cord injury. J Clin Endcr Metab 2003; 88: 402-407.

38 Singh R, Rohilla RK, Saini G, Kaur K. Longitudinal study of body composition in spina cord injury patients. Indian J Orthop 2014; 48: 168

39 Taittonen M, Raty H, Kirvela O, Aantaa R, Kanto J. The metabolic effects of oral tizanidine in healthy volunteers. Acta Anaesthesiol Scand 1995; 39: 628-632.

40 Gorgey AS, Caudill C, Sistrun S, Khalil RE, Gill R, Castillo T et al. Frequency of dietary recalls, nutritional assessment, and body composition assessment in men with chronic spinal cord injury. Arch Phys Med Rehabil 2015; 96: 1646-1653.

41 Harris JA, Benedict FG. A Biometric Study of Basal Metabolism in Man. Carnegie Institution of Washington, Washington, WA, USA, 1919.

42 Quebbeman EJ, Ausman RK. Estimating energy requirements in patients receiving parenteral nutrition. AMA Arch Surg 1982; 117: 1281-1284.

43 Spanier AH, Shizgal HM. Caloric requirements of the critically ill patient receiving intravenous hyperalimentation. Am J Surg 1977; 133: 99-104.

44 Cunningham JJ. A reanalysis of the factors influencing basal metabolic rate in normal adults. Am J Clin Nutr 1980; 33: 2372.

45 Schofield WN. Predicting basal metabolic rate, new standards and review of previous work. Hum Nutr Clin Nutr 1985; 39: 5-41.

Supplementary Information accompanies this paper on the Spinal Cord website (http://www.nature.com/sc) 